This is the accepted version of the following article: [Wenbiao Zhang, Yongpan Cheng, Chao Wang, Wuqiang Yang and Chi-Hwa Wang, Investigation on Hydrodynamics of Triple-Bed Combined Circulating Fluidized Bed Using Electrostatic Sensor and Electrical Capacitance Tomography, Industrial \& Engineering Chemistry Research, 52 (32), 2013, pp 11198-11207], which has been published in final form at [DOI: 10.1021/ie4009138]. In addition, authors may also transmit, print and share copies with colleagues, provided that there is no systematic distribution of the submitted version, e.g. posting on a listserve, network or automated delivery.

\title{
Investigation on Hydrodynamic of Tripe-bed Combined Circulating Fluidized Bed using Electrostatic Sensor and Electrical Capacitance Tomography
}

\author{
Wenbiao Zhang ${ }^{1,2}$, Yongpan Cheng ${ }^{1}$, Chao Wang ${ }^{2}$, Wuqiang Yang ${ }^{3}$ and Chi-Hwa Wang ${ }^{1, *}$ \\ 1. Department of Chemical and Biomolecular Engineering, National University of Singapore, 4 \\ Engineering Drive 4, Singapore 117576, Singapore \\ 2. School of Electrical Engineering and Automation, Tianjin University, Tianjin 300072, China \\ 3 School of Electrical and Electronic Engineering, The University of Manchester, Manchester M13 \\ 9PL, UK
}

*Correspondence should be addressed to: Chi-Hwa Wang, Tel: 65-6516-5079. Fax: 65-6779-1936.

E-mail: chewch@nus.edu.sg

\begin{abstract}
Coal gasification is used to convert coal into electricity, hydrogen and other valuable energy products, with reduced emission of $\mathrm{CO}_{2}$. To improve the energy conversion efficiency and further reduce the pollutant emission, Triple-bed combined Circulating Fluidized Bed (TBCFB) was proposed for coal gasification. Despite of the negative effects of electrostatics, it is useful for characterizing the hydrodynamics in a fluidized bed. Combined with an Electrical Capacitance Tomography (ECT) system, a novel electrostatic sensor has been fabricated to monitor the flow dynamics in the riser and downer of the TBCFB. By cross correlating signals from two corresponding electrostatic sensors in the upstream and downstream of the fluidized bed, the particle velocities can be obtained. The experimental results show an annular solids distribution and nearly flat particle velocity distribution near the inlet of the riser. In the downer, the solids distribution is inhomogeneous and the average velocity of the cross section is higher than the local velocities near the wall. All these results prove that the electrostatic sensor and ECT can be used to characterize the hydrodynamics in the fluidized bed.
\end{abstract}

Keywords: Fluidization; Electrical Capacitance Tomography; Electrostatic Sensor; Hydrodynamic; Circulating Fluidized Bed.

\section{Introduction}

Coal gasification is used to convert coal into electricity, hydrogen and other valuable energy products with reduced emission of $\mathrm{CO}_{2}$, which is a major contributor to climate change and global warming. ${ }^{1}$ 
Triple-Bed combined Circulating Fluidized Bed (TBCFB) is a relatively new and emerging technology that provides an efficient method for low-cost energy with low emission and environmental impacts. In fluidization processes and granular flow systems, tribo-electrification is inevitable due to the collisions, friction and rolling between particles, fluid and wall. This phenomenon may result in undesirable particle agglomeration and affects the hydrodynamics of the system and fluidization process efficiency. ${ }^{2}$ Tribo-electrification may also result in inaccuracy in the measurement as well as malfunction of instruments. Excessively charged particles tend to discharge in the form of fire and explosion, which poses safety risks in industry. Therefore, it is challenging but of profound theoretical and practical significance to systematically investigate the electrostatic phenomena in gas-solids fluidized beds.

In the past few decades, research has been done on the tribo-electrification phenomena and its effect on the flow dynamics in gas-solid fluidized beds. Reduction of electrostatic charge accumulation by increasing the humidity of fluidizing gas was investigated by Park et al. ${ }^{3}$ They found that the increase in the relative humidity of the fluidizing air to $40-80 \%$ could reduce the accumulation of electrostatic charge, increase the surface conductivity, and enhance charge dissipation. To understand the influence of fine particles on generation and dissipation of electrostatic charge, Mehrani et al. ${ }^{4}$ performed experiments in a Faraday cup fluidization column. The results showed that the charges on smaller particles were opposite to those on the larger particles. The effect of particle size of polyethylene resin on electrostatic charge generation and reactor wall fouling was analyzed by Sowinski et al. ${ }^{5}$ It was found that the smaller particles had a higher charge and resulted in more reactor wall fouling. Moughrabiah et al. ${ }^{6}$ examined the effects of pressure, temperature and gas velocity on electrostatics in gas-solids fluidized beds. It was found that with the increase in pressure or air velocity, the generation of electrostatic charges was increased due to more frequent particle-particle, particle-wall collisions and increased bubble rise velocity and flow rate, With the increase in temperature, the charge generation was reduced for the limited range of conditions studied.

In addition to experimental study, numerical simulation was widely applied to investigate the electrostatics phenomenon. Al-Adel et al. ${ }^{7}$ emphasized the importance of considering electrostatic effects for analyzing gas-solids flows of radial segregation of particles in vertical risers. Their model involving the effects of an electric field captured important qualitative features of riser flows: core-annular particle distribution, annular particle down-flow at low riser gas velocities, and annular up-flow at high gas velocities. Rokkam et al. ${ }^{8}$ developed an electrostatic model, which coupled with a multi-fluid computational fluid dynamic (CFD) model to analyze the electrostatic effect on polymerization fluidized bed reactors. Discrete Element Method (DEM) was adopted by Cheng et al. ${ }^{9}$ to simulate generation and transfer of electrostatic charge occurring in a downer.

Although electrostatics can cause many problems in industry, it can be utilized to measure the hydrodynamics in a gas-solids flow system. The electrostatic signal generated contains information about the flow dynamics. Matsusaka et al. ${ }^{10}$ used two short pipes that were made of different materials to simultaneously measure the mass flow rate and charge-to-mass ratio of particles in a gas-solids flow. Xu et al. ${ }^{11}$ used power spectrum analysis and Hilbert-Huang transform to the electrostatic 
fluctuations to extract and characterize the intrinsic features of dense-phase gas-solids flows. Portoghese et al. ${ }^{12}$ developed tribo-electric probes for real-time measurement of moisture content in fluidized beds. The tribo-electric signals were correlated with the moisture contents determined by titration for calibration, which was found to be sensitive to moisture contents below $100 \mathrm{ppm}$. As a non-intrusive low-cost technique, Electrical Capacitance Tomography (ECT) has been widely used in monitoring the performance of fluidized beds. ${ }^{13-17}$ This paper presents a novel electrostatic senor for measurement of the particle velocity and electrostatic distribution in the riser and downer of Triple-Bed combined Circulating Fluidized Bed. The reconstructed images by ECT can reflect the solids distribution in a cross sectional. Combined the information obtained from the electrostatic sensor and ECT together, the hydrodynamic of Triple-Bed combined Circulating Fluidized Bed can be revealed.

\section{Experimental setup and sensors}

\subsection{Experimental setup}

Fig. 1 shows the lab-scale Triple-Bed Circulating Fluidized Bed, which is made of acrylic, as a cold-model experimental setup. It can be used for coal gasification and is composed of a riser $(40 \mathrm{~mm}$ I.D. and $4 \mathrm{~m}$ high), a downer (40 mm I.D. and $2 \mathrm{~m}$ height), a gas-solid separator and a Bubbling Fluidized Bed (BFB) $(150 \mathrm{~mm} \times 300 \mathrm{~mm} \times 700 \mathrm{~mm})$. Air compressor is used to supply compressed air (about 5\% relative humidity) to the bottom of the riser. Polyethylene (PE) particles with a density of $941 \mathrm{~kg} / \mathrm{m}^{3}$ and average sdiameter of $2.7 \mathrm{~mm}$ are used as test material. Gravity forces the PE particles to flow down to the bottom of the riser from the bubbling fluidized bed. Then, compressed air blows up the particles along the riser. At the top of the riser, the solid particles pass through a smooth elbow into cyclone for gas-solids separation. As a result, the air escapes from the top of the cyclone, and the particles flowe down to the distributor above the downer. The gas and solids move down along the downer, and gas/solids separation takes place at the end of the downer by a separator and entrains solid particles to return to the BFB. A piece of gauze netting is used at the bottom of the downer to measure the solids mass flux, by measuring the weight of the collected particles. With a given collection time, the solids mass flux can be calculated. The superficial gas velocity ranges from 12.6 to $15.9 \mathrm{~m} / \mathrm{s}$.

\subsection{AC-based Electrical Capacitance Tomography (AC-ECT)}

An AC-ECT system from ECT Instruments Ltd (UK) is based on measuring the disparities in capacitance as a result of the variations in the electrical permittivity or distribution of the materials inside a vessel. The AC-ECT system is a non-invasive tool for online monitoring of fluidized beds. Wang et al. ${ }^{16,17}$ used such system to study the bubbling and slugging fluidized beds and fluidized bed dyers, which showed good performance. The AC-ECT system includes an AC-ECT frontend unit, a data acquisition (DAQ) board and a host PC. In comparison to an old generation of ECT system (charge/discharge ECT system developed in the early 1990s), the AC-ECT system has much improved sensitivity and high resolution (up to $0.01 \mathrm{fF}$ ) and it is not influenced by electrostatic effects. Another 
improvement in the AC-ECT system is the data acquisition rate, 300 frames/second with an 8-electrode sensor and 140 frames/ second with a 12-electrode sensor.

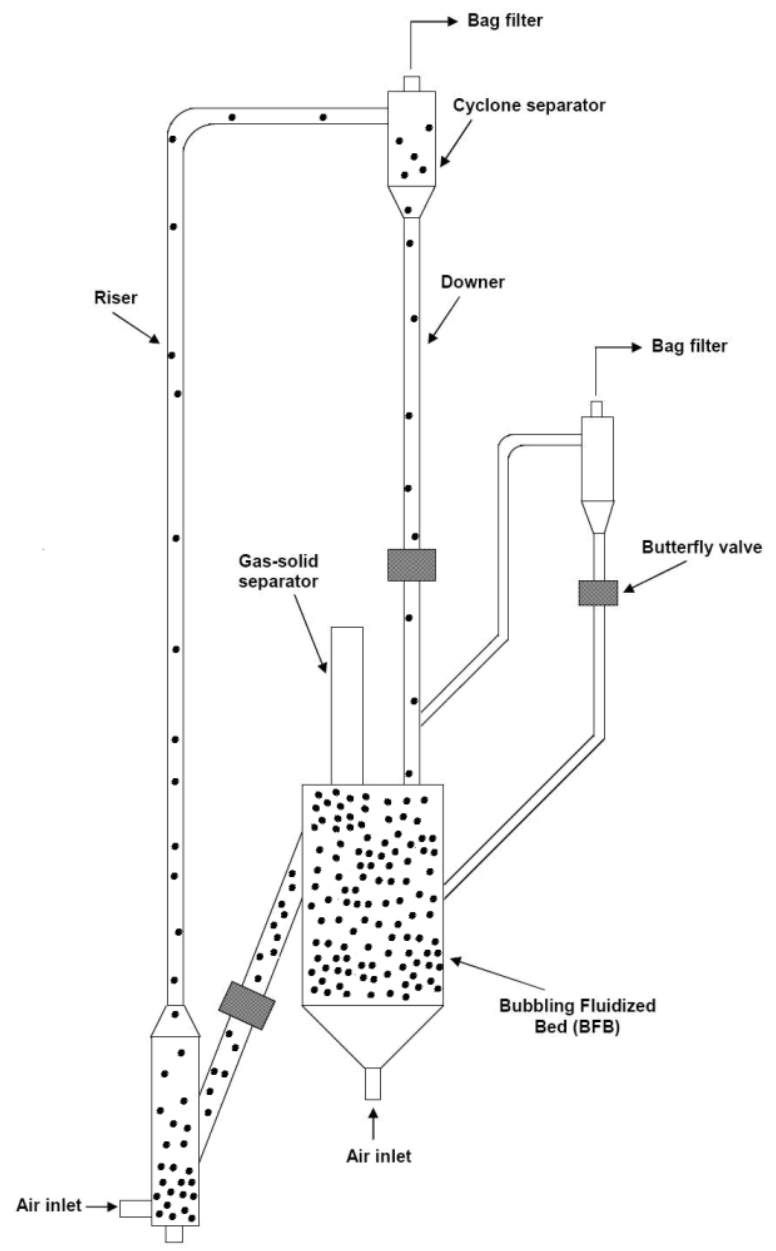

Figure 1: Lab-scale Triple-Bed Circulating Fluidized Bed as cold model experimental setup for coal gasification

An ECT sensor with a number of electrodes mounted around a vessel to measure the changes in capacitance between pairs of electrodes. The number and length of the electrodes, diameter of the sensor and the size of earthed screens are the main factors in designing ECT sensors. ${ }^{18}$ As shown in Figure 2 the ECT sensor used in this study consists of a cylindrical outer array of 8 copper electrodes and two earthed copper screens. The 8 electrodes are mounted on the outer wall of the chamber. The dimensions of the ECT sensor are given in Table 1. In this work, both Linear Back Projection (LBP) and Landweber algorithms are used for image reconstruction. The Maxwell model is employed to estimate capacitance.

Table 1: Dimensions of ECT sensor

\begin{tabular}{ll}
\hline Length of sensor & $320 \mathrm{~mm}$ \\
Diameter of frame (chamber) & $50 \mathrm{~mm}$ \\
Dimensions of electrodes & $200 \times 15.6 \mathrm{~mm}$ \\
Distance between electrodes & $4 \mathrm{~mm}$ \\
Distance between electrodes and earthed screens & $1 \mathrm{~mm}$ \\
\hline
\end{tabular}




\begin{tabular}{ll}
\hline Relative electrical permittivity of air & 1.0006 \\
Relative electrical permittivity of PE & $2.2-2.5$ \\
\hline
\end{tabular}

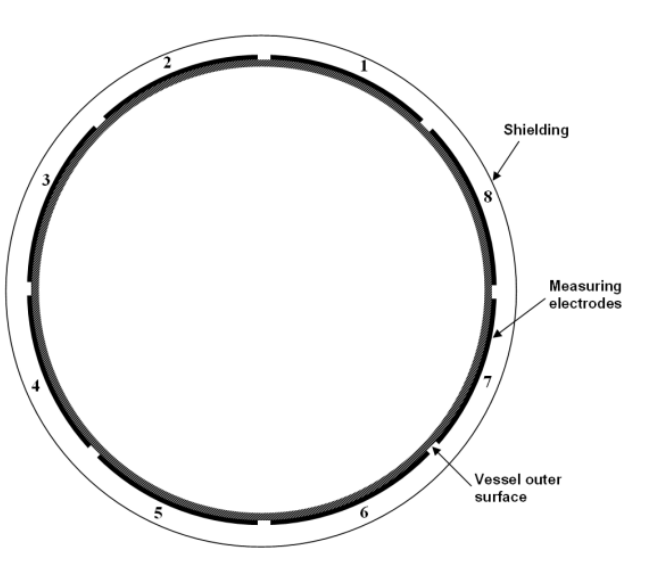

(a) Cross-section

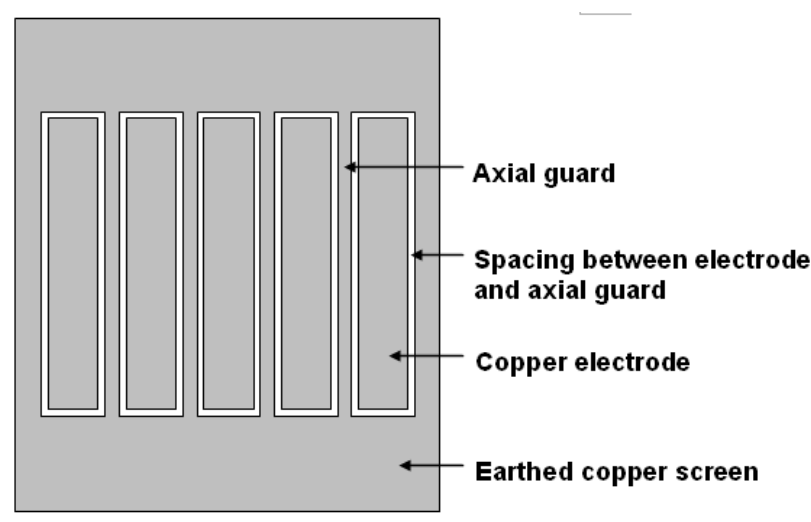

(b) Electrodes

Figure 2: ECT sensor with 8 electrodes.

\subsection{Electrostatic sensor}

Electrostatic charges is almost unavoidable in a fluidized bed, due to the repeated particle contacts, separation and friction of particles rubbing against each other and the wall. The resulted electrostatic signal contains information about the flow dynamics of the fluidized bed, which can be used to monitor the bed performance. A novel electrostatic sensor is presented in this paper. The senor had 2 ring-shape electrodes and 8 arc-shape electrodes as shown in Figure 3. The 10 electrodes are classified into five groups, one ring-shape group (A) and four arc-shape groups (B, C, D, E). The distance between the up-stream electrode and down-stream electrode is $50 \mathrm{~mm}$. The detailed ring-shape electrode and arc-shape electrode is also shown in Figure 3. The electrodes made of copper are closely wrapped around the outside of the pipe. The axial width and the thickness of each electrode were 6 $\mathrm{mm}$ and $2 \mathrm{~mm}$. Signal conditioning circuits has been developed. The electrodes and circuits are shielded in a metal box to eliminate noise.

To analyze the performance of the electrostatic sensor, the sensitivity distributions has to be obtained. The electrostatic field due to the particles' charge distribution in a pipe is determined by Poisson's equation:

$$
\nabla^{2} \bullet \Phi=-\frac{\rho}{\varepsilon_{0}}
$$

Where $\nabla^{2}$ is the Laplace operator, $\Phi$ is the electric potential, $\rho$ is the charge density and $\varepsilon_{0}$ is the permittivity of free space. After solving the electric potential $\Phi$, the electric field $E$, surface charge density $\sigma$ can be found from the relation:

$$
\sigma=\varepsilon_{0} E=-\varepsilon_{0} \bullet \nabla \Phi
$$




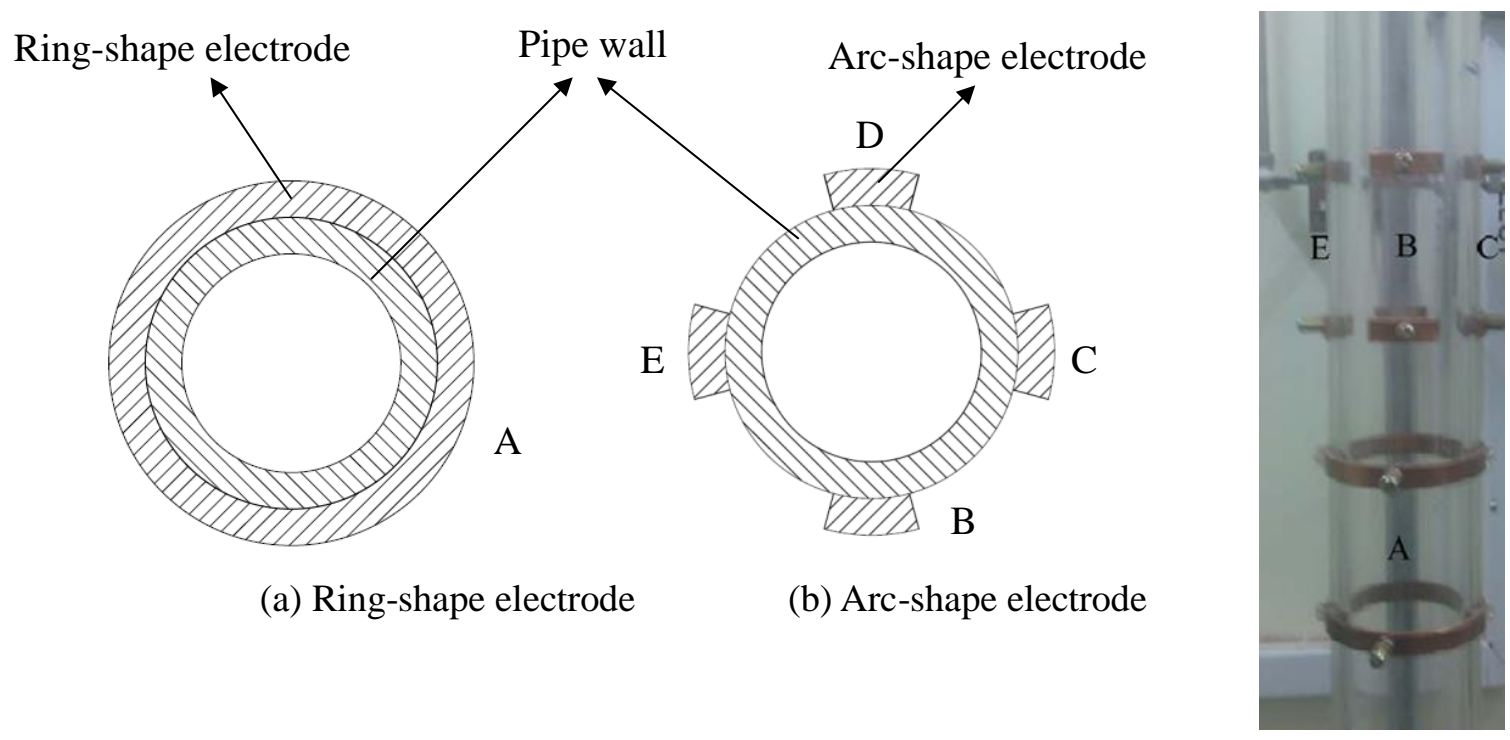

(c) Photo

Figure 3: Electrostatic sensor with ring-shape electrodes and arc-shape electrodes

The quantity of induced charge $Q$ on the inner surface of the electrode is given by:

$$
Q=\int_{s} \sigma d s
$$

The sensitivity of the electrode in one position is defined as:

$$
S=Q / Q_{p}
$$

Where $Q_{p}$ is the charge on the particles.

The sensitivity distributions of the ring-shape electrodes and arc-shape electrodes can be obtained using a model built in COMSOL. The COMSOL model of arc-shape electrodes is shown in Figure 4, with only one arc-shape electrode. Tetrahedral quadratic Lagrange elements are used in the mesh mode of the model. The mesh for the domain near the electrode is much finer than other sub-domains so as to reflect the charge distribution in the inner face of the electrode clearly.

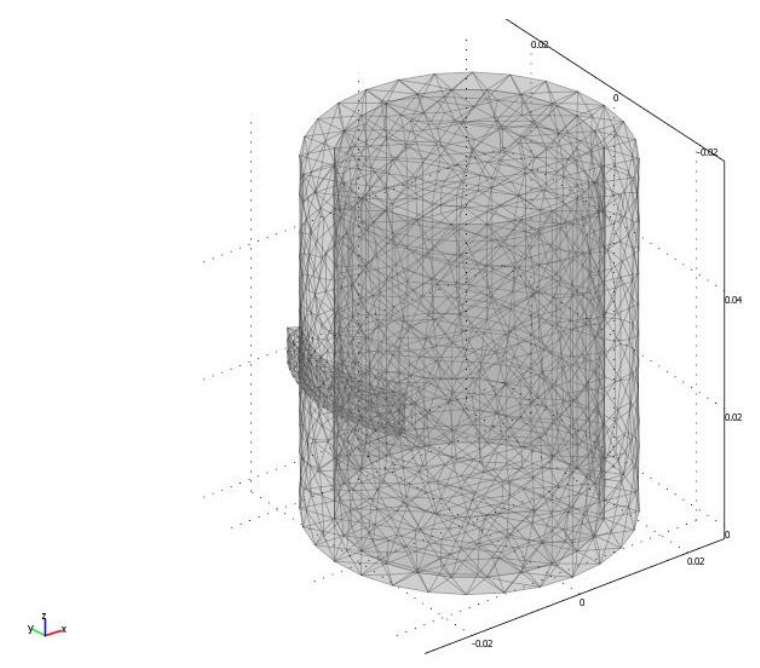

Figure 4: COMSOL model of arc-shape electrode

The materials of the pipe and electrode are acrylic and copper respectively. A point charge of $1 \mathrm{C}$ is used to model the single charged particle. The sensitivity distribution of arc-shape electrode is shown in Figure 5 (a). It can be seen that the sensitivity zone of arc-shape electrode is localized. The 
arc-shape electrode is only sensitive to the charged particles near the electrode.

The sensitivity distribution of ring-shape electrode is shown in Figure 5 (b). Although the sensitivity is little higher near the wall, the ring-shape electrodes reflected the average information of the flow dynamics in the cross section. It means that using this new sensor, the average information of solid particles in the cross section and the particles' local information near the wall can be obtained.

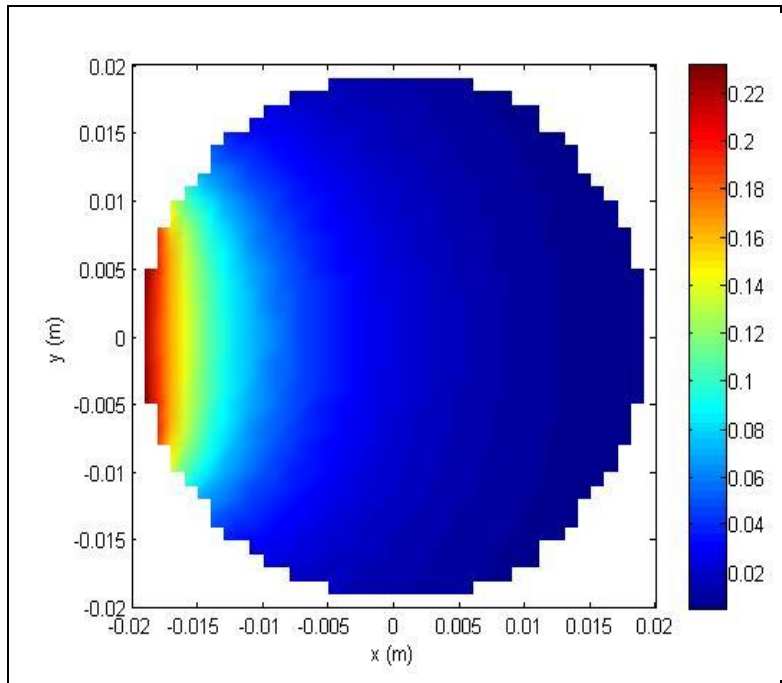

(a) Arc-shape electrode

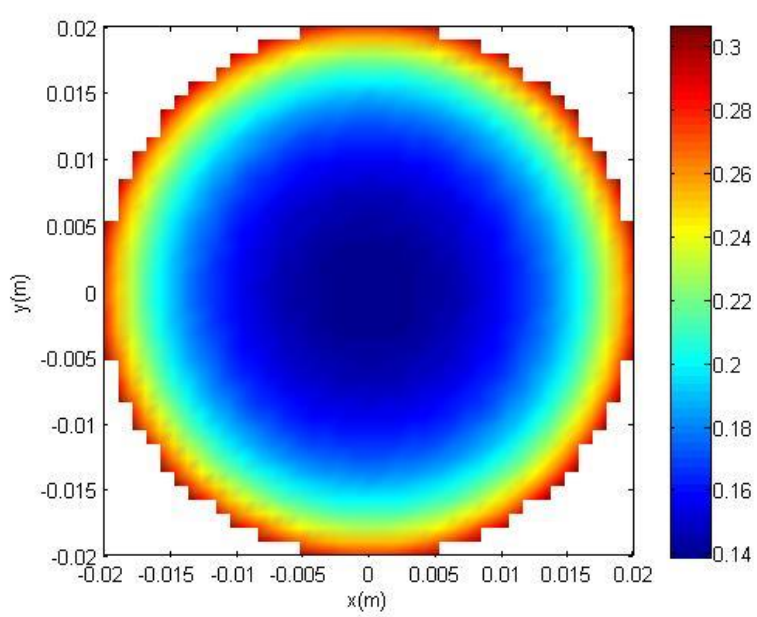

(b) Ring-shape electrode

Figure 5: Sensitivity distribution

When the charged particles move near the electrodes, induced charges are generated on the electrodes. Duo to the virtual earth on the signal conditioning circuit, there is induced current on the electrodes. It is integrated, filtered and amplified to generate a voltage signal proportional to the induced charges on the electrodes. The voltage signals from the electrodes are amplified and then sampled by a data acquisition module NI USB 6016 using LabVIEW. The 10 electrodes in the sensor are sampled simultaneously. The sampling rate is $20 \mathrm{kHz}$ and the sampling time under each flow rate is $60 \mathrm{~s}$. The typical sampled signals from the up-stream and down-stream ring-shape electrodes are shown in Figure 6. The two signals show similar trends with a time delay.

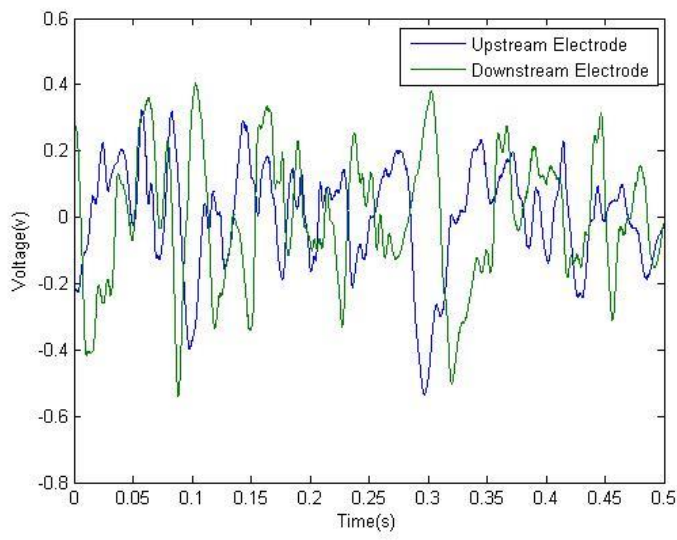

Figure 6: Sampled signals from up-stream and down-stream ring-shape electrodes 
The cross-correlation function is given by:

$$
R_{X Y}(\tau)=\frac{1}{T} \int_{0}^{T} y(t)_{X}(t-\tau) d t
$$

Where $\mathrm{x}(\mathrm{t})$ and $\mathrm{y}(\mathrm{t})$ are up-stream and down-stream signals and $T$ is the integration time. The delay corresponding to the maximum of correlation function is the time delay between the up-stream and down-stream electrodes, from which the velocity can be calculated. In this study, the signals from the five groups of electrodes are cross-correlated to obtain the velocity. The number of samples used for correlation is 10,000 , which corresponds to the integration time $T$ of $0.5 \mathrm{~s} .120$ correlation velocities are obtained from one group of electrodes under each flow rate. If the coefficient is too low, the calculated result does not present an acceptable particle velocity. The correlation velocity is discarded if the coefficient is less than 0.6 , as proposed by Nieuwland et al. ${ }^{19}$ The standard deviations (SD) of electrostatic signals from the arc-shape electrodes are computed, which reflect the magnitude of charge fluctuations on different electrodes of the wall and can be used to analyze the electrostatic distribution of solid particles.

\section{Experiments}

\subsection{In the riser}

Experiments were conducted in the riser part of the Triple-Bed Combined Circulating Fluidized Bed with the electrostatic sensor and ECT the sensor. The ECT sensor and the electrostatic sensor are mounted above the inlet, $150 \mathrm{~mm}$ and $550 \mathrm{~mm}$ respectively. By changing the superficial air velocity, the hydrodynamics in the riser can be changed.

The measured velocities from different groups of electrodes under different air velocities are given in Figure 7. The standard deviation of the velocities from the ring-shape electrodes is shown as error bar. Four groups of arc-shape electrodes were used to measure the local solids velocity near the pipe wall and one group of ring-shape electrodes used to measure the average solids velocity in the cross section. It can be seen that with the increase in air velocity, the average solid particle velocity and local solid particle velocity near the wall increase. As shown by the standard deviation of the velocities from the ring-shape electrodes, the velocities from the ring-shape electrodes and the velocities from four groups of arc-shape electrodes are similar, presenting a flat velocity profile.

Figure 9 shows ECT images with the air velocity being $13.8 \mathrm{~m} / \mathrm{s}$. The percentage value in the color bar shows the relative solids holdup in the cross section calculated from the reconstructed image, (100\% represents the solids packing value, nearly 0.63 ). From Figure 8 it can be seen that the solids distribution is annular in the lower part of the riser. 

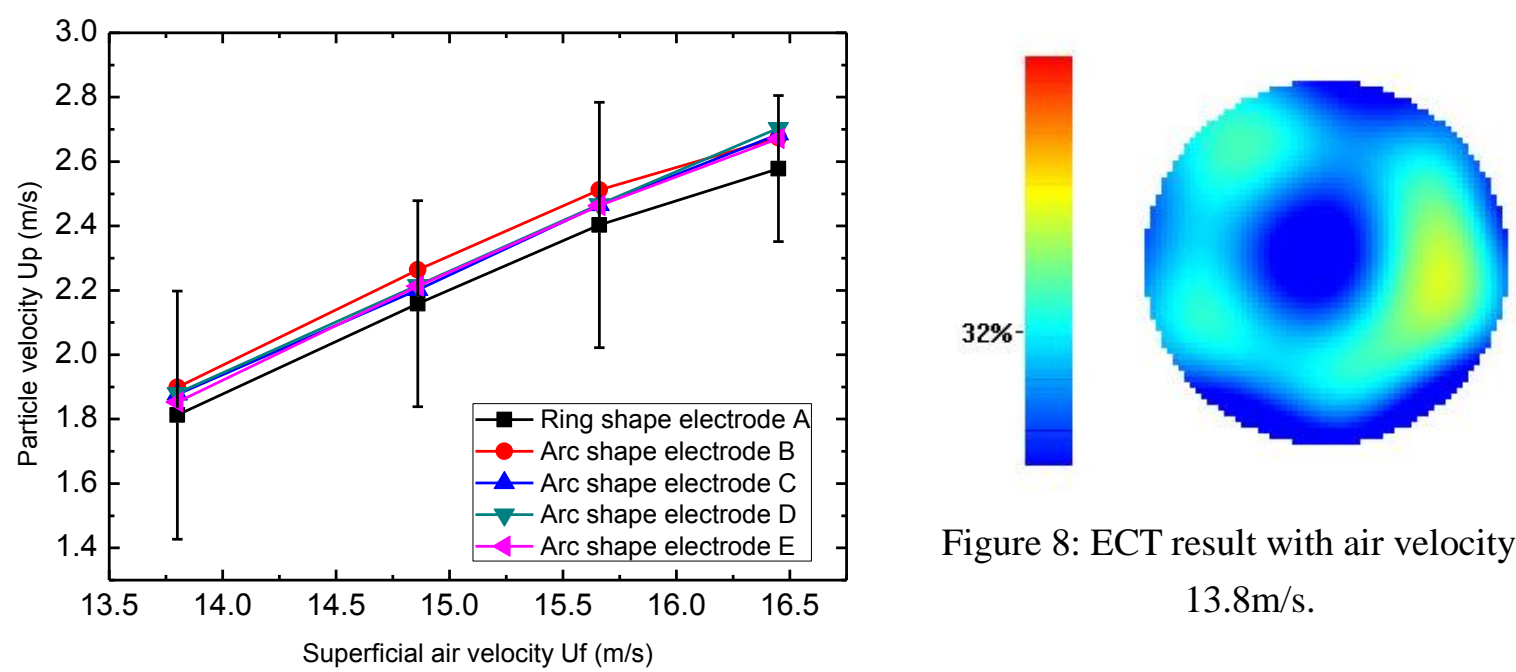

Figure 8: ECT result with air velocity of $13.8 \mathrm{~m} / \mathrm{s}$.

Figure 7: Velocities from different groups of electrodes

Table 2 gives the standard deviations of electrostatic signals from the up-stream arc-shape electrodes in the riser. SD_B, SD_C, SD_D, SD_E represent the standard deviations of electrostatic signals from group B, C, D, E up-stream arc-shape electrodes. It can be seen that with the increase in air velocity, the standard deviation of the signals increases. The reason for this is that with the increase in air velocity, the solids mass flux also increases, and there would be higher possibility for tribo-electrification, resulting in the increase in charges on the particles. From the reconstructed images by ECT it can be seen that it is an annular solids distribution, the solids holdup near the wall is nearly the same. As a result, the standard deviations of different electrodes given in Table 2 are similar.

Table 2 Standard deviation of electrostatic signals from up-stream arc-shape electrodes in the riser

\begin{tabular}{|c|c|c|c|c|}
\hline Air velocity & SD_B & SD_C & SD_D & SD_E \\
\hline 16.45 & 0.149 & 0.144 & 0.143 & 0.144 \\
\hline 15.66 & 0.146 & 0.141 & 0.139 & 0.139 \\
\hline 14.86 & 0.130 & 0.131 & 0.13 & 0.127 \\
\hline 13.8 & 0.115 & 0.112 & 0.115 & 0.11 \\
\hline
\end{tabular}

\subsection{In the downer}

Experiments were also conducted in the downer part of the Triple-Bed Combined Circulating Fluidized Bed with the electrostatic sensor and the ECT sensor. The ECT sensor and the electrostatic sensor are mounted above the inlet of the downer $1650 \mathrm{~mm}$ and $1250 \mathrm{~mm}$ respectively. By increasing the air velocity in the riser, the solids mass flux in the downer would also increase. A bypass air velocity in the downer (lower than $0.3 \mathrm{~m} / \mathrm{s}$ ), as the drag force acting on the solid particles is usually lower than $1 / 10$ of gravity, and so the solids velocity in the downer is close to the free fall velocity under gravity.

Figure 9 shows the velocities from different groups of electrodes in the downer under different air 
velocities in the riser. The standard deviation of the velocities from the ring-shape electrodes is shown as error bars. With the increase in air velocity in the riser, the bypass air velocity in the downer increases, resulting in the increase in the velocity measured from the electrostatic sensor. Comparing the error bars in Figure 8 and Figure 9, it can be found that in the downer, the velocities from the ring-shape electrodes present smaller fluctuations, indicating more stable flow condition. Near the inlet of the riser, the solid particles are in the acceleration zone and even backflow can happen occasionally. However, the solid particles in the downer behave like free fall under gravity. In the downer the flow condition is more stable.

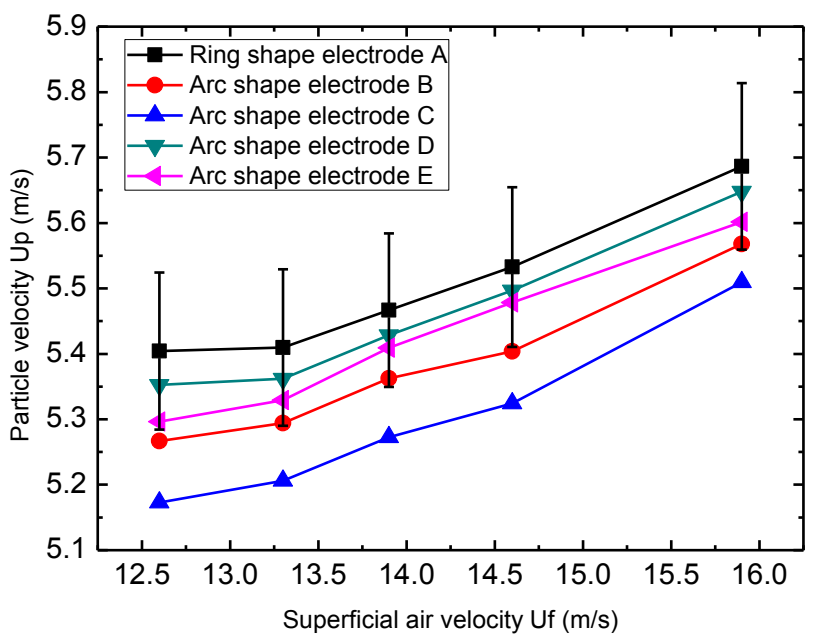

Figure 9: Velocities from different groups of electrodes in the downer

The difference in the velocities from different groups of electrodes can be obviously seen in Figure 10, which shows inhomogeneous velocity distribution in the downer. The average velocity from the ring-shape electrodes is higher than the local velocity from the arc-shape electrodes. Figure 10 shows the ECT results in the downer under different solid mass flow rates. It shows that with the increase in solid mass flux, the solid holdup in the cross section increases. It is noted that a pipe bend connected from the top of the riser to the cyclone above the downer generates inhomogeneous solids distribution after the bend, as captured by the reconstructed ECT images. The differences in the local velocities from different groups of arc-shape electrodes may also come from the inhomogeneous solids distribution in the downer.

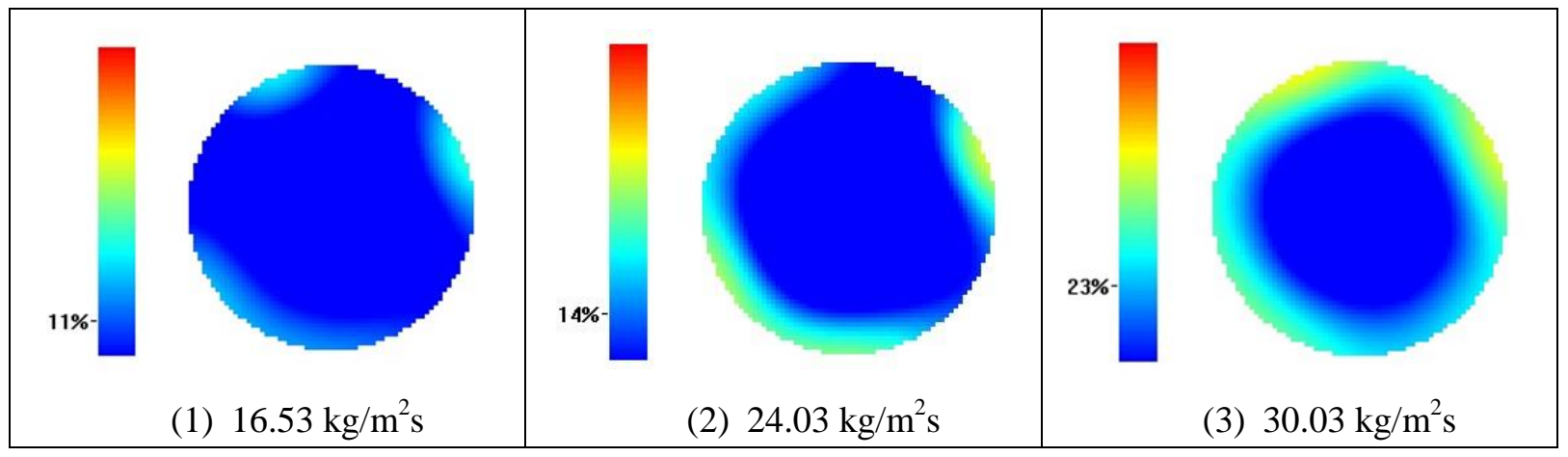

Figure 10: ECT results in the downer with different solids mass flux 
Table 3 showed the standard deviation of electrostatic signals from the up-stream arc-shape electrodes in the downer. With the increase in solids mass flux in the downer, the standard deviation of the arc-shape electrodes increases, indicating the increase in electrostatic charges on different sides of the wall. The electrostatic distribution is inhomogeneous due to the inhomogeneous solids distribution in the downer.

Table 3 Standard deviation of electrostatic signals from up-stream arc-shape electrodes in the downer

\begin{tabular}{|l|c|c|c|c|}
\hline $\begin{array}{l}\text { Solids mass } \\
\text { flux in downer } \\
\left(\mathrm{kg} / \mathrm{m}^{2} \mathrm{~s}\right)\end{array}$ & SD_B & SD_C & SD_D & SD_E \\
\hline 13.42 & 0.137 & 0.146 & 0.142 & 0.128 \\
\hline 16.53 & 0.153 & 0.161 & 0.154 & 0.14 \\
\hline 18.47 & 0.156 & 0.161 & 0.159 & 0.145 \\
\hline 24.03 & 0.162 & 0.17 & 0.167 & 0.154 \\
\hline 30.03 & 0.166 & 0.176 & 0.175 & 0.159 \\
\hline
\end{tabular}

\section{Conclusion}

A novel electrostatic sensor is introduced to characterize the hydrodynamics in the circulating fluidized bed. The ring-shape electrodes can give average information of solid particles in a cross section, and the arc-shape electrodes can give local information on solids flow dynamics near the pipe wall. Using the electrostatic sensor and ECT, the hydrodynamic of Tripe-Bed combined Circulating Fluidized Bed is investigated. An annular solids distribution and nearly flat particle velocity distribution present near the inlet of the riser. In the downer, the solids distribution is inhomogeneous and the average velocity in the cross section is higher than the local velocities near the wall. The flow is more stable in the downer than in the riser. From the experimental results, it can be concluded that the electrostatic sensor and ECT can be used to monitor the hydrodynamics of fluidized beds.

\section{References}

(1) Guan, G.Q. Fushimi, C.; Ishizuka, M.; Nakamura, Y.; Tsutsumi, A.; Matsuda, S.; Suzuki, Y.; Hatano, H.; Cheng, Y.P.; Lim, E.W.C.; Wang, C. H. Flow behaviors in the downer of a large-scale triple-bed combined circulating fluidized system with high solids mass fluxes. Chem. Eng. Sci., 2011, 66, 4212-4220.

(2) Mehrani, P; Bi, H.T.; Grace, J.R. Electrostatic charge generation in gas-solid fluidized beds. J. Electrost. 2005, 63, 165-173.

(3) Park, A. H.; Bi, H.T.; Grace, J.R. Reduction of electrostatic charges in gas-solid fluidized beds. Chem. Eng. Sci., 2002, 57, 153-162.

(4) Mehrani, P; Bi, H.T.; Grace, J.R. Electrostatic behavior of different fines added to a Faraday cup 
fluidized bed. J. Electrost. 2007, 65, 1-10.

(5) Sowinski, A; Mayne, A.; Mehrani, P. Effect of fluidizing particle size on electrostatic charge generation and reactor wall fouling in gas-solid fluidized beds. Chem. Eng. Sci., 2012, 71, $552-563$

(6) Moughrabiah, W.O.; Grace, J.R.; Bi, X. T. Effects of pressure, temperature, and gas velocity on electrostatics in gas-solid fluidized beds. Ind. Eng. Chem. Res. 2009, 48, 320-325.

(7) Al-Adel, M.F.; Saville, D.A.; Sundaresan, S. The effect of static electrification on gas-solid flows in vertical risers. Ind. Eng. Chem. Res. 2002, 41, 6224-6234.

(8) Rokkam, R.G.; Fox, R.O.; Muhle, M.E. Computational fluid dynamics and electrostatic modeling of polymerization fluidized-bed reactors. Powder Technol. 2010, 203, 109-124.

(9) Cheng, Y. P.; Lau, Y.J. Guan, G.Q.; Fushimi, C.; Tsutsumi, Wang, C. H. Experimental and Numerical Investigations on the Electrostatics Generation and Transport in the Downer Reactor of a Triple-Bed Combined Circulating Fluidized Bed. Ind. Eng. Chem. Res. 2012, 51, 14258-14267.

(10) Matsusaka, S.; Masuda, H. Simultaneous measurement of mass flow rate and charge-to-mass ratio of particles in gas-solids pipe flow. Chem. Eng. Sci., 2006, 61, 2254-2261.

(11) Xu, C.L.; Liang, C.; Zhou, B.; Wang, S.M. HHT analysis of electrostatic fluctuation signals in dense-phase pneumatic conveying of pulverized coal at high pressure. Chem. Eng. Sci., 2010, 65, 1334-1344.

(12) Portoghese, F.; Berruti, F.; Briens, C. Continuous on-line measurement of solid moisture content during fluidized bed drying using triboelectric probes. Powder Technol. 2008, 181, 169-177.

(13) Chaplin, G.; Pugsley, T. Application of electrical capacitance tomography to the fluidized bed drying of pharmaceutical granule. Chem. Eng. Sci., 2005, 60, 7022-7033.

(14) Du, B.; Warsito, W.; Fan, L.S. Imaging the choking transition in gas-solid risers using electrical capacitance tomography. Ind. Eng. Chem. Res. 2006, 45, 5384-5395.

(15) Liu, S.; Chen, Q.; Wang, H.G.; Jiang, F.; Ismail, I; Yang, W.Q. Electrical capacitance tomography for gas-solids flow measurement for circulating fluidized beds. Flow measurement and instrumentation, 2005, 16, 135-144.

(16) Wang, H.G.; Yang, W.Q.; Senior, P.; Raghavan, R.S.; Duncan, S.R. Investigation of batch fluidized-bed drying by mathematical modeling, CFD simulation and ECT measurement. AIChE Journal, 2008, 54, 427-444.

(17) Wang, H.G.; Yang, W.Q.; Dyakowski, T.; Liu, S. Study of bubbling and slugging fluidized beds by simulation and ECT. AIChE Journal, 2006, 52, 3078-3087.

(18) Yang, W.Q. Design of electrical capacitance tomography sensors. Measurement Science and Technology 2010, 21, art. no. 042001.

(19) Nieuwland, J.J.; Meijer, R.; Kuipers, J.A.M.; van Swaaij, W.P.M. Measurement of solid concentration and axial solids velocity in gas-solid two phase flow. Powder Technol. 1996, 87, 127-139. 